\title{
FORMAÇÃO PROFISSIONAL DE OBSTETRIZES E ENFERMEIRAS OBSTÉTRICAS: VELHOS PROBLEMAS OU NOVAS POSSIBILIDADES?
}

\author{
MARIA LUIZA GONZALEZ RIESCO
}

Universidade de São Paulo

MARIA ALICE TSUNECHIRO

Universidade de São Paulo

\begin{abstract}
Resumo: Refere-se às transformações na formação profissional de parteiras, obstetrizes e enfermeiras obstétricas no Brasil, desde a criação dos cursos de parteiras vinculados às escolas médicas, no século XIX, até as mais recentes experiências, mediante cursos de especialização em enfermagem obstétrica. Discute os modelos de formação dos profissionais que existem em outros países, considerando tanto o ensino independente da obstetrícia como a modalidade vinculada aos cursos de enfermagem. Apresenta proposta de um curso de obstetrícia para ser oferecido por escolas de enfermagem.
\end{abstract}

Palavras-chave: obstetriz, enfermagem obstétrica, ensino.

Este artigo trata da formação de obstetrizes e enfermeiras obstétricas no Brasil, além de traçar um pequeno panorama em outros países, para fornecer elementos de compreensão dos diferentes modelos que existem.

As profissões de enfermeira, parteira, obstetriz e enfermeira obstétrica, em sua origem, formação e exercício profissional, são diferenciadas. Parteira é o título mais antigo dessa profissional, posteriormente denominada enfermeira obstétrica e obstetriz. Enfermeira obstetra é a denominação mais recente e consolida a formação de enfermeira (substantivo), adjetivada pela titulação de especialista na área (obstetra, como adjetivo). Embora pareçam nuanças de menor importância, traduzem modificações na legislação de ensino e na concepção quanto à modalidade de formação e da própria profissão.'

Com relação à capacitação profissional de obstetrizes e enfermeiras obstétricas, historicamente coexistem dois tipos de programas educacionais: o modelo europeu, ou via direta, com o ingresso em cursos de obstetrícia anexos às escolas médicas ou de enfermagem, e o americano, em que a habilitação formal na área de obstetrícia é 
concebida como uma especialidade da enfermagem. Diversos países mantêm essas duas modalidades de formação, e o modelo americano representa uma tendência mundial. ${ }^{2} \mathrm{~A}$ via direta tradicionalmente é a mais encontrada na Europa, mas tem sido objeto de interesse crescente em vários países. ${ }^{3}$

No Brasil, no século XIX, a educação formal de parteiras iniciou-se junto às escolas médicas, que controlaram sua formação até meados do século $X X$. O primeiro documento legal sobre o ensino de parteiras data de 1832, quando as Academias Médico-Cirúrgicas do Rio de Janeiro e da Bahia foram transformadas em Faculdades de Medicina e, entre os cursos oferecidos, foi incluído o Curso de Partos. Até então, as mulheres que se dedicavam a partejar deveriam possuir uma 'carta de examinação', concedida pelo Físico-Mor ou Cirurgião-Mor do Império, e uma licença da Chancelaria. Mesmo que essa lei não fosse obedecida, representou um princípio de controle sobre o exercício da atividade das parteiras pelos médicos. De 1832 até 1949, toda a legislação do ensino de parteiras esteve contida na legislação do ensino da medicina. No período, a legislação sobre o ensino de parteiras foi objeto de diversos decretos que determinaram a denominação dos cursos, as exigências para admissão de candidatas, o currículo a ser seguido e o título conferido parteira, enfermeira parteira, enfermeira especializada, obstetriz e enfermeira obstétrica. A denominação de enfermeira especializada para a parteira começou a ser usada nos anos de 1920 e a de obstetriz apareceu pela primeira vez como o título conferido às formadas no Curso de Obstetrícia da Faculdade de Medicina e Cirurgia do Pará, entre 1922 e 1925. Em 1955, reapareceu em texto legal que regulamentava o exercício da enfermagem profissional e distinguia a obstetriz das demais categorias, ou seja, enfermeiro, auxiliar de enfermagem, parteira, enfermeiro prático ou prático de enfermagem e parteira prática. ${ }^{4}$

Nas mudanças do ensino de parteiras, observou-se um crescimento das exigências para admissão, seja em idade mínima, seja em conhecimentos das Ciências Humanas, Biológicas e Exatas, além da regulamentação do número de horas de estágio. Outra particularidade foi o Decreto Federal $n^{\circ} 7.247$, de 5 de abril de 1879 , que possibilitava 0 ingresso de alunos do sexo masculino, embora o curso tenha sido freqüentado exclusivamente por mulheres. ${ }^{5}$

Com relação ao ensino de enfermagem no país, em 1890 , foi criada no Rio de Janeiro a Escola Profissional de Enfermeiras e Enfermeiros no Hospício Nacional dos Alienados e, no início do novo século, foram organizadas outras escolas particulares e públicas em vários estados brasileiros. Entre elas, destaca-se a Escola Dona Anna Nery, em 1923, no Rio de Janeiro, cujo modelo de ensino baseado no sistema norte-americano foi considerado padrão.

A associação do trabalho da parteira e da enfermeira foi sendo cogitada já no final do século XIX, quando médicos brasileiros passaram a propor a formação profissional de parteiras que fossem também enfermeiras. Com essa perspectiva, buscavam-se mudanças na atuação das parteiras, limitando sua prática independente, restringindo e controlando seu espaço na assistência ao parto e impondo a hierarquia estabelecida para as enfermeiras, especialmente no hospital.

\footnotetext{
${ }^{2}$ Esse modelo de formação é mais recente e iniciou-se nos Estados Unidos da América e no Brasil nas décadas de 1920 e 1940, respectivamente. Ver Dilce JORGE, 1975; e Judy LITOFF, 1982.

${ }^{3}$ Essa modalidade, denominada direct entry model, no Canadá e na Inglaterra vem substituindo a formação de enfermeiras obstétricas, a partir de 1980. Ver Michael KLEIN, 1994; Louise SILVERTON, 1996; e Dawna VANWYCK, 1992.

${ }^{4}$ JORGE, 1975.

${ }^{5}$ Ver JORGE, 1975.
} 
As principais modificações na legislação do ensino de parteiras, no início do século $X X$, relacionam-se à extinção dos cursos de parteiras e à criação de cursos de enfermeiras de maternidades anexos às faculdades de medicina. Assim, em 1925, o curso de parteiras da Faculdade de Medicina do Rio de Janeiro foi encerrado. Em 1927, o currículo da Escola de Parteiras de São Paulo sofreu alterações e a disciplina Enfermagem Geral foi incluída no programa. Em 1931, a escola passou a denominar-se Escola de Obstetrícia e Enfermagem Especializada, o currículo foi direcionado ao ensino da enfermagem e o curso passou a ter a duração de três anos; ao seu final, as alunas obtinham, além do título de Parteira, o de Enfermeira Especializada. ${ }^{6}$

Em 1939, em São Paulo, foi fundado o Curso de Enfermagem Obstétrica anexo à Clínica Obstétrica da Escola Paulista de Medicina, tendo formado apenas uma turma. A exigência legal para admissão das candidatas ao curso limitava-se ao ensino primário e era de curta duração, com dois anos. O médico Álvaro Guimarães Filho, professor responsável pelo curso, observou que essa formação era precária e propôs a criação da especialização em Obstetrícia. Assim, as alunas deveriam iniciar o curso com formação em enfermagem geral e, nos últimos três semestres, cursar as cadeiras especializadas em Obstetrícia. $^{7}$

A partir de 1949, as modificações, que já vinham ocorrendo na prática, acabaram sendo incorporadas à legislação. As escolas de enfermagem passaram, oficialmente, a formar enfermeiras obstétricas. A portadora de diploma de enfermeira podia freqüentar 0 Curso de Especialização em Enfermagem Obstétrica e, ao final de um ano, receber o certificado de enfermeira obstétrica.

Os dois tipos de formação eram oferecidos, e os cursos de enfermagem e de obstetrícia funcionavam de maneira independente. Com a crescente hospitalização do parto, o campo de atuação das parteiras passou a ser objeto de disputa entre estas, as enfermeiras e os médicos, e uma grande polêmica foi gerada entre enfermeiras e parteiras. De um lado, as enfermeiras não aceitavam que as parteiras formadas pelos médicos nos cursos anexos às clínicas obstétricas das faculdades de medicina recebessem o título de enfermeira obstétrica e lutavam por consolidar o curso como uma especialidade da enfermagem. Para elas, os cursos de parteira, denominados de enfermagem obstétrica, eram um exemplo de especialização sem base, uma vez que a formação anterior em enfermagem não era exigida das candidatas. ${ }^{8}$ Por outro lado, as parteiras argumentavam que no mundo inteiro o ensino da obstetrícia era responsabilidade de médicos, professores da clínica obstétrica, e que a enfermagem e a obstetrícia eram profissões afins, porém distintas, não sendo possível conferir às enfermeiras com um ano de especialização as mesmas competências e prerrogativas asseguradas àquelas que faziam o curso de obstetrícia.

Na década de 1960, o Conselho Federal de Educação estabeleceu uma solução conciliatória ao fixar um novo currículo, com duração de três anos para os cursos de obstetrícia, estabelecendo a articulação com o curso de enfermagem por meio de um

\footnotetext{
${ }^{\circ}$ Currículo do curso iniciado em 1931: $1^{a}$ série: Anatomia, Fisiologia e Higiene; Microbiologia; Química e Nutrição; Farmacologia; $2^{a}$ série: Patologia e Enfermagem Elementar; Enfermagem Médica; Enfermagem Cirúrgica; Enfermagem Obstétrica; $3^{a}$ série: Enfermagem Obstétrica; Enfermagem Ginecológica; Enfermagem do Recém-Nascido; História e Ética. Regulamento da Escola de Obstetrícia e Enfermagem Especializada de São Paulo. São Paulo, Tip. Mário Giorgis, s.d. Ver: Maria Lúcia MOTT, 2002.

7 Álvaro GUIMARÃES FILHO, 1941.

${ }^{8}$ Anayde CARVALHO, 1976.
} 
tronco profissional comum de dois anos e um terceiro ano diversificado. Essa proposta representou a possibilidade de complementação, com mais um ano de curso em Enfermagem ou em Obstetrícia para obstetrizes e enfermeiras, respectivamente.

No início da década de 1970, foi introduzida outra modificação decorrente da reformulação das universidades brasileiras, que propunha "vedar a duplicação de meios para fins idênticos ou equivalentes". Em função da similaridade entre os currículos de enfermagem e de obstetrícia, os cursos foram fundidos em três fases sucessivas: 1) a préprofissional; 2) o tronco profissional comum que levava à graduação do enfermeiro; 3) e as habilitações que levavam à formação da enfermeira obstétrica ou obstetriz, do enfermeiro médico-cirúrgico e do enfermeiro de saúde pública. As duas primeiras etapas tinham duração mínima prevista de 2.500 horas, integralizadas entre três e cinco anos, e o ciclo completo, com a habilitação em uma das áreas, de no mínimo 500 horas, passava para 0 total de 3.000 horas, cursadas no período de quatro a seis anos. ${ }^{9}$ A partir de 1972 , coube exclusivamente às escolas de enfermagem a formação profissional da enfermeira obstétrica ou obstetriz, como única via para a capacitação formal de não-médicos para assistência ao nascimento e ao parto normal.

Assim, com a incorporação dos cursos de obstetrícia às escolas de enfermagem e a fusão dos currículos, a opção deixou de existir no ingresso à universidade, sendo postergada para o último ano do curso, na dependência da oferta de vagas para essa habilitação. Embora o curso tivesse a denominação legal de Enfermagem e Obstetrícia, gradativamente, menos escolas ofereciam essa formação, reduzindo o contingente de profissionais no mercado de trabalho.

Em 1994, o currículo mínimo de enfermagem foi modificado e as habilitações foram extintas. Na atualidade, o único curso previsto para formação específica de profissionais não-médicos na área obstétrica consiste na especialização em enfermagem, nível de pós-graduação lato sensu.

No âmbito internacional, as entidades que representam obstetrizes e enfermeiras obstétricas - Confederação Internacional de Obstetrizes (International Confederation of Midwives - ICM) e Conselho Internacional de Enfermeiras (International Council of Nurses $I C N$ ) - elaboraram um documento preparatório ${ }^{10}$ para uma reunião realizada com a finalidade de analisar tendências no ensino, definir políticas conjuntas para a formação e o exercício de obstetrizes e enfermeiras e delimitar territórios profissionais. Nesse documento, constam as seguintes definições:

Obstetriz (Midwife): pessoa que, tendo sido regularmente admitida em programa educacional de obstetrícia, legalmente reconhecido no respectivo país, concluiu e foi aprovada no programa de estudos de obstetrícia e adquiriu os requisitos de qualificação para ser registrada ou legalmente licenciada para exercer a obstetrícia. A definição corresponde à adotada pela ICM e pela Federação Internacional de Ginecologia e Obstetrícia (FIGO), considerada uma das mais amplamente aceitas em nível internacional.

Enfermeira obstétrica (Nurse Midwife): pessoa que é legalmente licenciada ou registrada para exercer todas as atribuições de enfermeira e de obstetriz em seu país. A qualificação em obstetrícia pode ser obtida prévia ou posteriormente à qualificação em enfermagem ou como uma combinação dos ensinos de enfermagem e obstetrícia. Essa é a definição seguida pelo ICN.

\footnotetext{
${ }^{9}$ Resolução nº 4/1972 do Conselho Federal de Educação, que trata do currículo mínimo dos cursos de Enfermagem e Obstetrícia. Ver JORGE, 1975.

${ }^{10} \mathrm{~A}$ Discussion Paper - Nurses and Midwifery, elaborado pelo Conselho Internacional de Enfermeiras (ICN), 1994(MID/ 95/FAA/3).
} 
Auxiliar de obstetriz (Auxiliary Midwife): pessoa que recebeu algum treinamento formal em obstetrícia e atua sob critérios e supervisão direta ou indireta da obstetriz. O período de formação pode variar de um a dois anos. Em alguns casos, a pessoa pode ter recebido qualificação como auxiliar de enfermagem; em outros, ensino que combina enfermagem e obstetrícia. Auxiliares de obstetrícia podem ou não ser licenciadas. Elas podem receber o título de obstetriz rural, assistente de obstetriz, etc.

Parteira tradicional (Traditional Birth Attendant): pessoa que assiste a mãe durante o parto e que, inicialmente, adquiriu habilidades no atendimento solitário da parturiente ou como aprendiz de outra parteira tradicional. A parteira tradicional recebe um breve curso de treinamento em algum serviço de saúde atualizado para aprimorar seus conhecimentos. O período de treinamento costuma não se estender além de um mês, embora possa se alongar por mais tempo. Podem ser conhecidas como parteiras leigas, etc.

A referida reunião visou também a atender a demandas produzidas por iniciativas da Organização Mundial da Saúde (OMS), como o Projeto Safe Motherhood, estabelecendo, assim, parcerias em projetos de intervenção de interesse comum.

A OMS, no Projeto Safe Motherhood, privilegia o desenvolvimento de recursos humanos e refere-se textualmente à prioridade na capacitação de parteiras nos diversos níveis de escolaridade. A avaliação desse projeto, realizada em 1995, em diversos países, aponta a essencialidade do desenvolvimento de recursos humanos para atingir os objetivos dos programas. " Para a OMS, a saúde reprodutiva insere-se na concepção de saúde como parte do processo de desenvolvimento humano. Assim, as propostas da OMS para capacitação profissional na área vêm trazendo, como marco conceitual, a ampliação do enfoque de risco, introduzindo as perspectivas de gênero, sexualidade, integralidade biopsicossocial e participação social como elementos essenciais. Outro princípio considerado essencial para o ensino e a prática da saúde reprodutiva refere-se à responsabilidade social, cujos elementos significativos são a ética, a eqüidade, o respeito pela autodeterminação e o ambiente humano. Esses aspectos estão contidos no documento da Organização Pan-Americana da Saúde, intitulado Marco de referência para la enseñanza de la salud reprodutiva, ${ }^{12} \mathrm{com}$ propostas relativas às instituições formadoras, ao processo de formação, a conteúdos, à metodologia e à avaliação. ${ }^{33}$

Para ilustrar a situação em diferentes regiões do mundo, no quadro abaixo estão agrupados os dados relativos à modalidade de formação profissional da auxiliar de obstetriz, da obstetriz e da enfermeira obstétrica em 132 países.

\footnotetext{
1 TECHNICAL MEETING..., 1995.

12 PAN AMERICAN HEALTH ORGANIZATION, 1994.

${ }^{13} \mathrm{O}$ documento traz em anexo uma análise situacional do ensino da saúde reprodutiva nas Ciências da Saúde, no Brasil, assinalando que não existe uma estrutura normatizada, sistematizada para seu ensino, que tem enfoque predominantemente biologicista. Os aspectos demográficos dão ênfase aos índices e não ao processo. Os temas principais são discutidos em disciplinas isoladas (Medicina Preventiva, Ginecologia, Obstetrícia, Pediatria, Doenças Transmissíveis) e sem integração, com conseqüente fragmentação do currículo; a atenção primária em saúde é pouco discutida nos currículos e o ensino não está apoiado na realidade social.
} 
QUADRO - Exercício mundial da obstetrícia

\begin{tabular}{|c|c|c|c|}
\hline $\mathrm{Y}_{\mathrm{LOCAL}}$ & $\begin{array}{l}\text { AUXILIAR DE } \\
\text { OBSTETRIZ } \\
\text { n. }{ }^{\circ} \text { de países }\end{array}$ & $\begin{array}{c}\text { OBSTETRIZ } \\
\mathrm{n} \cdot{ }^{\circ} \text { de países }\end{array}$ & $\begin{array}{c}\text { ENFERMEIRA OBSTÉTRICA } \\
\text { n. }{ }^{\circ} \text { de países }\end{array}$ \\
\hline $\begin{array}{l}\text { ÁSIA } \\
\text { (24 países) }\end{array}$ & 12 & 11 & 21 \\
\hline $\begin{array}{l}\text { ORIENTE MÉDIO } \\
\text { (15 países) }\end{array}$ & - & 10 & 6 \\
\hline $\begin{array}{l}\text { OCEANIA } \\
\text { (16 países) }\end{array}$ & 1 & 3 & 13 \\
\hline $\begin{array}{l}\text { ÁFRICA } \\
\text { (23 países) }\end{array}$ & 2 & 10 & 15 \\
\hline $\begin{array}{l}\text { EUROPA } \\
\text { ( } 31 \text { países) }\end{array}$ & - & 19 & 14 \\
\hline $\begin{array}{l}\text { AMÉRICAS E CARIBE } \\
\text { (23 países) }\end{array}$ & - & 9 & 19 \\
\hline
\end{tabular}

Fonte: A Discussion Paper - Nurses and Midwifery, ICN, 1994.

Nos diferentes países, é preciso considerar que os pré-requisitos para a admissão ao curso e sua duração variam, conforme a realidade e a legislação de ensino de cada local. Existem cursos de auxiliar de obstetriz com duração de seis meses a quatro anos e com exigência de escolaridade prévia que pode chegar a dez anos. Os cursos de obstetriz podem ter diferentes graus de escolaridade em um mesmo país, como, por exemplo, a obstetriz de saúde pública ou comunitária e a obstetriz generalista ou sênior. A duração dos cursos varia nos diversos países, de 18 meses a 4 anos, após escolaridade básica que pode chegar a 11 anos. Para as enfermeiras obstétricas, a diversidade é muito maior, havendo uma sucessão de combinações para a qualificação formal. Existem currículos integrados, em que a formação é concomitante ao curso de enfermagem, e países onde a enfermeira só é qualificada na área em nível de pós-graduação.

No entanto, há posições contrárias que consideram o ensino compartilhado para estudantes de obstetrícia e enfermagem prejudicial. $O$ argumento é que os estudantes de obstetrícia com freqüência não têm uma idéia clara de sua identidade profissional e submetem-se ao viés da enfermagem, mais voltada para os aspectos patológicos do processo saúde-doença. ${ }^{14}$

A experiência do Canadá é recente e bastante significativa do ponto de vista da reabilitação profissional da parteira. No início do século passado, as parteiras canadenses foram suplantadas por médicos e enfermeiras, e uma pequena minoria, qualificada fora do país, continuou atuando clandestinamente em regiões desprovidas de serviços de saúde.

\footnotetext{
${ }^{14}$ SILVERTON, 1996.
} 
Na década de 1990, a profissão ressurgiu em algumas províncias - Alberta, Colômbia Britânica, Manitoba, Ontário e Saskatchewan - em razão das elevadas taxas de intervenções obstétricas e do lobby de organizações de feministas contra a medicalização do parto e os aspectos paternalistas na assistência à saúde da mulher. No sentido de capacitar parteiras e legalizar seu trabalho, o caminho seguido pelo Canadá revelou dois aspectos que merecem destaque: o debate em torno da autonomia profissional da parteira e a modalidade de formação. Os principais questionamentos expressam preocupações com a reação corporativa de médicos e enfermeiras, dado que as parteiras canadenses são formadas pelo direct entry model, desvinculado da enfermagem, constituindo um grupo independente. Essas 'novas' parteiras vêm perfilando sua inserção no sistema de saúde e, rejeitando o modelo médico na assistência ao parto, têm "mostrado o quanto foi equivocado ter proscrito as parteiras como um anacronismo"..$^{15}$

No Brasil, a elevada morbimortalidade materna e perinatal e o número excessivo de operações cesarianas oneram o sistema do ponto de vista financeiro e social e produzem uma crise na assistência obstétrica. Nesse contexto, a questão da capacitação de recursos humanos para assistência ao nascimento e ao parto tem sido objeto de estudos ${ }^{16} \mathrm{e}$ debates, gerando propostas alternativas e envolvendo enfermeiras, obstetrizes, médicos, formuladores de políticas públicas de saúde, educadores e organizações da sociedade civil, como associações profissionais, de usuárias e consumidoras dos serviços de saúde.

Um exemplo é o Projeto de Lei do deputado Eduardo Jorge que dispõe sobre o exercício da profissão de técnico em obstetrícia, prevendo a instituição do Curso Técnico em Obstetrícia Humanizada. ${ }^{17}$ As origens do Projeto situam-se nas exigências legais para a revalidação do diploma de Angela Gehrke, parteira formada na Alemanha com marcante atuação em São Paulo, assistindo partos em uma população favelada, a partir de meados da década de $1980 .{ }^{18}$

A proposta de criação do Técnico de Obstetrícia tem produzido polêmica no âmbito da enfermagem, gerando resistência e posicionamentos contrários nos fóruns e entidades da categoria, com argumentos, em geral, tão pouco consistentes quanto aqueles expressos na justificação do Projeto. De um lado, nega-se a proposição de um 'novo' profissional não-médico com atribuição de assistir o parto no lugar da enfermeira, da técnica e da auxiliar de enfermagem; de outro lado, faz-se a defesa de uma parteira com menor grau de escolaridade, mas com atribuições similares às da obstetriz e da enfermeira obstétrica. ${ }^{19}$

\footnotetext{
${ }^{15}$ BLAIS et al., 1994; Brian BURTCH e Carol HIRD, 1997; Tara EINES, 1993; KLEIN, 1994; VANWYCK, 1992.

${ }^{16}$ TSUNECHIRO, 1987; Ruth OSAVA, 1997; RIESCO, TSUNECHIRO e Isabel Cristina BONADIO, 1998; RIESCO e Rosa Maria FONSECA, 2002.

${ }_{17}$ Projeto de Lei n. ${ }^{\circ}$ 3.175/1997, do deputado Eduardo Jorge, de São Paulo, em tramitação na Câmara dos Deputados.

${ }^{18}$ Angela Gehrke atuou como parteira na Associação Comunitária Monte Azul, em São Paulo, sendo precursora do movimento pela humanização da assistência ao parto e fundadora de uma Casa de Parto naquela comunidade. Em 1999, após a complementação de estudos, o diploma de Angela foi revalidado como obstetriz pela Escola de Enfermagem Anna Nery da Universidade Federal do Rio de Janeiro. Angela adoeceu no mesmo ano, vindo a falecer em março de 2000, na Alemanha. Ver Sonia HOTIMSKY, 2001.

${ }^{19}$ Segundo Leda ASCHERMAN (1998), "os pontos críticos dessa proposta referem-se às seguintes questões: Como será a formação deste profissional? A quem estará ligado na equipe de saúde: à equipe médica, como antigamente, à equipe de enfermeiras obstétricas, ou a ninguém, atuando com autonomia técnica em casas de parto e longe de instituições hospitalares? O modelo de formação será padronizado ou serão respeitadas as características e peculiaridades regionais, considerando, inclusive, as parteiras tradicionais existentes? 'Importaremos' um modelo europeu, americano ou japonês de sucesso comprovado ou criaremos um 'da casa', com formas escorregadias, mas que permita uma atuação mais brasileira?".
} 
Diante da Reforma Sanitária em andamento no Brasil, o Ministério da Saúde organizou, em 1989, um Seminário Nacional para discutir a situação dos recursos humanos no Sistema Único de Saúde (SUS) e apontar diretrizes para a formulação de uma política de recursos humanos para a área da saúde. Entre as propostas de intervenção apresentadas no Seminário, destacam-se, no campo da administração de recursos humanos, os condicionantes para o planejamento e alocação, quais sejam: a) o quadro nosológico perfil epidemiológico; b) novo modelo assistencial; c) organização dos serviços, conforme os diversos níveis de complexidade. Por sua vez, a Lei Orgânica da Saúde que instituiu o SUS no país - Lei n. ${ }^{\circ} 8.080 / 1990$ - está pautada em diretrizes constitucionais e em obediência aos princípios de universalidade e igualdade de acesso aos serviços, como um direito de cidadania e dever do Estado. A referida lei prevê que o SUS atue na ordenação e formação de recursos humanos na área da saúde.

Desde 1999, o Ministério da Saúde vem financiando cursos de especialização em enfermagem obstétrica, mediante convênios firmados com universidades e secretarias de saúde em todo o país. Estes cursos são de carga horária reduzida, com no máximo 600 horas, com enfoque no ensino da assistência no pré-natal e no parto. ${ }^{20}$ Essa iniciativa constitui-se em um marco nacional no projeto de capacitação de enfermeiras para a assistência materna e perinatal, e em três anos aproximadamente 1.090 enfermeiras foram especializadas por esses cursos, com a grande maioria delas inserida em projetos locais de maternidades, centros de parto normal e programas de saúde da família.

Paralelamente, medidas políticas do Ministério da Saúde, como a inclusão do parto realizado por enfermeira obstétrica na tabela de pagamentos dos SUS e a instituição de centros de parto normal sob a coordenação exclusiva de enfermeiras obstétricas, têm produzido confrontos com a categoria médica. Essas medidas reacendem antigas disputas entre médicos e enfermeiras pelo direito de exercer a obstetrícia. Por sua vez, as mulheres, usuárias ou não do SUS, vêm manifestando sua insatisfação com a atenção médica que recebem durante o parto, o que favorece a inserção de obstetrizes e enfermeiras obstétricas nessa assistência.

Retomando a questão da capacitação de recursos humanos para assistência ao parto, há também polêmicas sobre qual deve ser a melhor opção para formar especialistas em enfermagem obstétrica. Os cursos mais acadêmicos e tradicionais, ${ }^{21}$ que tentam abranger todos os aspectos da assistência à saúde materna e da mulher e estimular a produção de conhecimentos, têm sido duramente criticados porque, além de despenderem muito tempo na formação da profissional e, conseqüentemente, recursos materiais e financeiros, não atendem à atual necessidade do quantitativo substancial de enfermeiras obstétricas prontas para atuar, especialmente no pré-natal, no parto e no pós-parto. Por sua vez, os cursos de especialização de 'curta duração', idealizados para atender à demanda por formação mais rápida, estão sendo direcionados quase que exclusivamente para a assistência ao parto, em detrimento de outros aspectos de atenção à saúde materna e da mulher. A modalidade de especialização continua sendo criticada, pois leva-se muito tempo para formar uma enfermeira e depois uma especialista em obstetrícia. ${ }^{22}$

Merece ser comentado que essas enfermeiras 'especialistas' não se sentem estimuladas a permanecer no campo da assistência ao parto. Estudo realizado com 92

\footnotetext{
20 Janine SCHIRMER, 2001.

${ }^{21}$ Esses cursos vinham sendo oferecidos por escolas de enfermagem até o final da década de 1990, tinham duração mínima de um ano e carga horária superior a 1.000 horas, com a finalidade de preparar profissionais para a prática assistencial e também para o ensino e a pesquisa.

22 OSAVA, 1997; TSUNECHIRO, RIESCO e BONADIO 1998; Nadia NARCHI, 2001.
} 
enfermeiras obstétricas, entre 202 formadas pela Escola de Enfermagem da Universidade de São Paulo, no período de 1980 a 1995, mostrou que 50\% delas não atuavam na área, o que sugere que a vinculação frágil com a especialidade seja um dos motivos para $o$ abandono da profissão. ${ }^{23}$ Essa dificuldade de se manter na área pode ter como causa uma identidade profissional híbrida, que favorece o 'acúmulo' ou o 'desvio de função' no exercício profissional, com o deslocamento da enfermeira obstétrica da assistência no pré-natal e no parto para outras áreas da enfermagem, gerando sobrecarga de trabalho, frustração, desmotivação, conformismo e submissão.

Embora a questão pedagógica da formação da parteira mereça estudos específicos, pode-se registrar que os conhecimentos necessários à parteira são provenientes da enfermagem, da medicina, da fisiologia, das ciências sociais, de outras parteiras, das mulheres, das pesquisas e do desenvolvimento de habilidades na comunicação e no relacionamento interpessoal, indispensável para dar suporte emocional. As mulheres, embora não constituam um grupo homogêneo, esperam que a obstetrícia praticada por parteiras esteja fundamentada nesses elementos. ${ }^{24}$

No Brasil, a atual legislação de ensino permite ampliar as propostas de formação de enfermeiras obstétricas. A Lei de Diretrizes e Bases da Educação Nacional configura-se como um instrumento flexível para a construção de novas opções no âmbito da educação superior, respeitando as possibilidades, finalidades e autonomia de cada instituição de ensino.

Assim, a proposição de um curso de graduação em obstetrícia com duração de três anos para formar obstetrizes seria uma alternativa à exclusiva especialização de enfermeiras. Há sugestões de formação de nível universitário, específica, comportando elementos da prática da enfermagem e da obstetrícia, distribuídos em curso de graduação de quatro anos, integrado às escolas de enfermagem. ${ }^{25} \mathrm{~A}$ opção pelo curso seria anterior ao ingresso, no momento do vestibular, caracterizando a via direta ou o denominado direct entry model, que forma a midwife (obstetriz, em inglês).

Essa formação seria bem conduzida pelas atuais escolas de enfermagem, que têm mantido os cursos de enfermagem obstétrica ao longo das últimas cinco décadas, como uma forma de resistência à extinção dessas profissionais, não obstante as inúmeras adversidades do mercado de trabalho e as limitações da legislação de ensino.

A formação da 'nova' obstetriz, condizente com os princípios que orientam o ensino de enfermeiras e enfermeiras obstétricas, daria maior ênfase aos aspectos fisiológicos, emocionais e socioculturais do processo reprodutivo, privilegiando um perfil de atuação fundamentado nas seguintes competências:

1. compreensão do fenômeno da reprodução como singular, contínuo e saudável, no qual a mulher é o foco central, e que se desenvolve em um determinado contexto sócio-histórico;

2. desenvolvimento do processo assistencial e educativo, com base na interação e parceria, possibilitando às pessoas envolvidas tomarem suas decisões de saúde;

3. articulação entre observações clínicas, conhecimento científico, habilidade técnica e julgamento intuitivo na tomada de decisões;

\footnotetext{
${ }^{23}$ Miriam MERIGHI, 2000.

${ }^{24}$ Mavis KIRKHAN, 1996.

${ }^{25}$ OSAVA, 1997.
} 
4. valorização do saber e da atuação interdisciplinares;

5. desenvolvimento das atribuições com base na responsabilidade ético-política e autonomia profissional.

A proposta apresentada vem sendo elaborada por docentes da Escola de Enfermagem da Universidade de São Paulo, visando à criação do curso de graduação em obstetrícia para formar obstetrizes pela via direta.

Finalmente, consideramos que as reflexões e proposições aqui sugeridas são contribuições ao debate, em consonância com as demandas atuais para a formação de enfermeiras obstétricas e obstetrizes e para as necessárias transformações do modelo assistencial na área da saúde materna e perinatal.

\section{REFERÊNCIAS BIBLIOGRÁFICAS}

ASCHERMAN, Leda. "A qualificação formal e os modelos alternativos de capacitação da equipe de enfermagem para assistência ao nascimento e parto". In: SEMINÁRIO ESTADUAL SOBRE O ENSINO DE ENFERMAGEM PARA A ASSISTÊNCIA AO NASCIMENTO E PARTO, $1 .$, 1998, Ribeirão Preto. Anais... Ribeirão Preto: Escola de Enfermagem de Ribeirão Preto da USP/Associação Brasileira de Obstetrizes e Enfermeiros Obstetras-São Paulo, 1998. p. 19-24.

BLAIS, Régis, MAHEUX Brigitte, LAMBERT Jean, LOISELLE Jacinhe, GAUTHIER Nathalie, and FRAMARIN Alicia. "Midwifery Defined by Physicians, Nurses and Midwives: the Birth of a Consensus." Canadian Medical Association, v. 150, n. 5, Mar. 1994. p. 691-697.

BURTCH, Brian; HIRD, Carol. "En pos del reconocimiento". Salud Mundial, v. 50, n. 2, p. 4-5, marzo/abr. 1997.

CARVALHO, Anayde C. Associação Brasileira de Enfermagem: 1926-1976. Brasília: Associação Brasileira de Enfermagem, 1976. Documentário.

EINES, Tara T. "Análisis del efecto de la legislación de salud del Canadá en el bienestar de la mujer". In: GÓMEZ, Elza G. (ed.). Género, mujer y salud en las Américas. Washington, OPS, 1993. Publicação científica n. 542, cap. 4, p. 245-257.

GUIMARÃES FILHO, Álvaro. "Aspectos sociais da assistência obstétrica". Revista de Obstetrícia e Ginecologia de São Paulo, v. 5, n. 3, p. 152-168, ago. 1941.

HOTIMSKY, Sonia N. Parto e nascimento no Ambulatório e na Casa de Partos da Associação Comunitária Monte Azul: uma abordagem antropológica. 2001. Dissertação (Mestrado)

- Faculdade de Saúde Pública da Universidade de São Paulo.

JORGE, Dilce R. Evolução da legislação federal do ensino e do exercício profissional da obstetriz (parteira) no Brasil. 1975. Tese (Livre-Docência) - Escola de Enfermagem Anna Nery da Universidade Federal do Rio de Janeiro.

KIRKHAN, Mavis. "Profissionalization Past and Present: with Women or with the Powers that be?" In: KROLL, Debra (ed.). Midwifery Care for the Future: Meeting the Challenge. London: Baillière Tindall, 1996. cap.9, p.164-201.

KLEIN, Michael C. "The Midwife Dossier: Cooperation or Competition?" LEditorial/ Canadian Medical Association, v. 150, n. 5, Mar. 1994. p. 657-660.

LITOFF, Judy B. 'The Midwifery Throughtout History". Journal of Nurse Midwifery, v. 27, n. 6, Nov./Dec. 1982. p. 3-11.

MERIGHI, Miriam Aparecida B. Enfermeiras obstétricas egressas da Escola de Enfermagem da Universidade de São Paulo: caracterização e trajetória profissional. 2000. Tese (LivreDocência) - Escola de Enfermagem da Universidade de São Paulo. 
MOT, Maria Lúcia. "As parteiras e a assistência ao parto em São Paulo nas primeiras décadas do século XX". Revista do Instituto Histórico Geográfico Brasileiro, 2002. No prelo

NARCHI, Nadia Z. "Aspectos legais do ensino e exercício profissional da enfermeira obstétrica: transformações e perspectivas". In: SEMINÁRIO ESTADUAL SOBRE O ENSINO DE ENFERMAGEM PARA A ASSISTÊNCIA AO NASCIMENTO E PARTO, 3., 2001 , São Paulo. Anais... São Paulo: Associação Brasileira de Obstetrizes e Enfermeiros Obstetras - Seção São Paulo e Escola de Enfermagem da Universidade de São Paulo, 2001. p. 93-101.

OSAVA, Ruth Hitomi. Assistência ao parto no Brasil: o lugar do não médico. 1997. Tese (Doutorado) - Faculdade de Saúde Pública da Universidade de São Paulo.

PAN AMERICAN HEALTH ORGANIZATION. Marco de referencia para la ensenãnza de la salud reprodutiva. Cartagena, 1994.

RIESCO, Maria Luiza G. "Enfermeira obstetra: herança de parteira e herança de enfermeira". Revista Latino-Americana de Enfermagem, v. 6, n. 2, p. 13-5, abr. 1998.

RIESCO, Maria Luiza G.; FONSECA, Rosa Maria G. S. "Elementos constitutivos da formação e inserção de profissionais não-médicos na assistência ao parto". Cadernos de Saúde Pública, v. 18, n. 3, p. 685-698, maio/jun. 2002.

RIESCO, Maria Luiza G.; TSUNECHIRO, Maria Alice; BONADIO, Isabel Cristina. "Obstetriz e enfermeira obstétrica: revendo sua formação". Acta Paulista de Enfermagem, v. 13, n. especial/II, p. 212-6, 2000.

SCHIRMER, Janine. Formação da enfermeira obstetra: contribuição para o resgate da atuação na assistência ao parto e nascimento. 2001. Tese (Livre-Docência) - Escola de Enfermagem da Universidade de São Paulo.

SILVERTON, Louise. "Educating for the Future." In: KROLL, Debra (ed.). Midwifery Care for the Future: Meeting the Challenge. London: Baillière Tindall, 1996. cap.5

TECHNICAL MEETING OF THE INTER-AGENCY GROUP FOR SAFE MATERHOOD. Issues in Essential Obstetric Care. New York: Measham, 1995.

TSUNECHIRO, Maria Alice. A formação da enfermeira obstétrica no Estado de São Paulo. 1987. Tese (Doutorado) - Escola de Enfermagem da Universidade de São Paulo.

TSUNECHIRO, Maria Alice; RIESCO, Maria Luiza G.; BONADIO, Isabel Cristina. "A qualificação formal e os modelos alternativos de capacitação da equipe de enfermagem para assistência ao nascimento e parto". In: SEMINÁRIO ESTADUAL SOBRE O ENSINO DE ENFERMAGEM PARA A ASSISTÊNCIA AO NASCIMENTO E PARTO, 1., 1998, Ribeirão Preto. Anais... Ribeirão Preto: Escola de Enfermagem de Ribeirão Preto da USP/Associação Brasilera de Obstetrizes e Enfermeiros Obstetras-São Paulo, 1998. p. 25-35

VANWYCK, Dawna M. "The 'New' Profession." Canadian Nurse, v. 88, n. 2, Feb. 1992. p. 1518.

\section{Midwifery and Nurse-Midwifery Education: Old Problems or New Possibilities?}

Abstract: This paper presents the historical transformation of midwifery and nurse-midwifery education in Brazil from the $19^{\text {th }}$ century until recent experiencies of training nurse-midwives as a graduate course in obstetrical nursing. It discusses the education models of these professionals in other countries and presents a proposal for midwifery education integrated into nursing schools.

Key words: midwife, nurse-midwifery, education. 